\title{
How did Children with Complex Care Needs Affect during the Lockdown Due to the COVID-19 Pandemic?
}

\author{
V Mundada 1, T Gupta ${ }^{2}$ A Pamfilo², A Joseph ${ }^{2}$ and S Banu ${ }^{2}$ \\ ${ }^{1}$ Aster DM Healthcare, Al Qusais, United Arab Emirates \\ ${ }^{2}$ Al Noor Training Centre for Persons with Disabilities, United Arab Emirates
}

\begin{abstract}
The COVID-19 pandemic and the lockdown imposed by most of the governments because of it have affected most of us including children with complex care needs. Because of various reasons like changed routine and social isolation, the physical and psychological health of these children has affected. With this observational study, we tried to assess new symptoms with which children with complex care needs presented during the lockdown period. We selected a cohort of children who usually attended 'Al Noor Training Centre for Persons with Disabilities' in Dubai, the United Arab Emirates where they received therapies but had to stay home and continue these therapeutic sessions through telemedicine. All new symptoms during these sessions were assessed by relevant specialists. Amongst the different symptoms which were mainly reported by the parents were sleep impairment, headache, behavioral issues, non-epileptic seizures, motor, and vocal tics. Identifying such symptoms in these children can be difficult, but it is important to manage them effectively with the help of professionals.
\end{abstract}

\section{Background}

Current pandemic of COVID-19 has significantly caused social, economic and health consequences across the world. Factors like social isolation and economic effects have also affected the mental and psychological health of many individuals. Children are also one of the vulnerable groups to get affected.

Children's clinical services and social activities have been disrupted in many countries as the result of the temporary closures of medical centres, schools, and caregiving agencies in the lockdown period. Children with neuro disabilities have faced additional challenges as a result of their functional limitation and changes to their daily routine [1]. For example, a child with autism may find it difficult to cope up with the new environment and exhibit behavioral symptoms like agitation. There could be added negative impact on the skill development due to suspension of speech therapy or occupational therapy like sessions. It is not uncommon for these children to present with somatic symptoms like headache or abdominal pain.

With this observational study, we wanted to understand the prevalence as well as the spectrum of new-onset symptoms that the children with complex care needs have been facing during the COVID-19 pandemic; mainly due to their disrupted structured routine including lack of ongoing support by the respective therapists as the result of lockdown and closure of medical facilities as well as special need schools.

\section{Methodology}

For this study, the cohort of children receiving telerehabilitation in 'Al Noor Training Centre for Persons with Disabilities' in Dubai, United Arab Emirates was chosen. This school caters the complex care needs of children with developmental and intellectual disabilities where children have access to various services like physiotherapy, occupational therapy, speech and language therapy and psychology.

Since March 2020 until September 2020, a total of 164 children who usually attend the school daily had been receiving telerehabilitation by various therapists depending on their ongoing care needs.

If any of the parents reported any new symptom, such symptoms were assessed by the respective specialists or the child was referred to the relevant physician or specialist for the formal assessment and management.

*Corresponding author: Dr. V Mundada, Consultant Pediatric Neurologist, Aster DM Healthcare, Medcare Women and Children Hospital, Dubai, United Arab Emirates, Tel: +971585950976

Accepted: March 16, 2021

Published online: March 18, 2021

Citation: Mundada V, Gupta T, Pamfilo A, et al. (2021) How did Children with Complex Care Needs Affect during the Lockdown Due to the COVID-19 Pandemic?. J Pediatr Neurol Neurosci 5(1):84-85

Copyright: () 2021 Mundada V, et al. This is an open-access article distributed under the terms of the Creative Commons Attribution License, which permits unrestricted use, distribution, and reproduction in any medium, provided the original author and source are credited. 


\section{Results}

Out of the total 164 children, 24 children were assessed for any new-onset symptoms. All these children had pre-existing intellectual disabilities. 11 children were diagnosed with autistic spectrum disorder while 5 were diagnosed with cerebral palsy and 2 children had Fragile $X$ syndrome. These new symptoms were either reported by the parents of the respective children or were observed by the therapists during the telerehabilitation. All the children who had new symptoms during this period were seen by the relevant specialists for the formal assessment and diagnosis of these symptoms. Those who were still waiting for the formal assessment and diagnosis of the new symptoms were excluded from this study.

All 24 children (100\%) had some form of sleep impairment. Two-third of them had sleep-onset association type and rest had limit-setting type behavioral insomnia. All of them were referred to the clinical psychologist after the diagnosis by the sleep medicine specialist.

The surge of behavioral problems was observed in 19 children (79\%). These included behavioral patterns like increased aggression, agitation, being violent towards family members and headbanging episodes. $4(16 \%)$ of these children needed evaluation by a psychiatrist while all of them were seen by the clinical psychologist. A third of these children were diagnosed to have some form of anxiety. Most of them (63\%) had Autistic spectrum disorder as the pre-existing diagnoses.

Next most common symptom was a headache. $63 \%$ had symptoms of headache. All these were reported by mainly the parents based on the observation. Following the assessment by the pediatric neurologist, all these children were diagnosed to have tension-type headaches, and none had any secondary type headache.

Two children (8\%) presented with new-onset seizures like episodes which were assessed by the pediatric neurologist. These were diagnosed as non-epileptic seizures. Both these children had pre-existing epilepsy. Similarly, new-onset motor tics were observed in two children (8\%) while vocal tics were seen in eight children (33\%). All these children were assessed by the pediatric neurologist and neuropsychologist.

\section{Discussion}

Widespread outbreaks of infectious diseases, such as COVID-19, are associated with psychological and mental health issues [2]. Children are not indifferent and are prone to the dramatic impact of the COVID-19 epidemic. Understanding their reactions and emotions is essential to address their needs properly. These could be challenging especially in the children with complex care needs. These children can have difficulty in understanding the complexity of the situation and are faced with missing ongoing learning and therapy support as the result of lockdown, even the parents.

There have been many published studies on the mental health consequences of children during COVID-19 pandemic. Many such children are presenting with various somatic symptoms like tension-type headaches, tics, non-epileptic seizures etc. Even children with complex care needs have been facing similar issues. The lockdown has known to cause negative effects on morale, behavior, and social interactions in these children. Although parents are performing therapies for such children at home, they could struggle to cope up as they often lack the professional expertise and usually rely on the support of their schools and therapists for any such issue [3].

With this study, we wanted to understand the physical issues that such children could be facing apart from their background physical and mental health needs. Such new symptoms could affect not only the children but their parents also.

Our result of this study has revealed that sleep is almost invariably affected in all the children of our cohort. Changed routine can be the reason causing such impairment. New symptoms like headache need to be carefully assessed instead of simply symptomatically treating or over investigating them. It is important to understand and address any such symptom effectively as there can be an underlying psychological issue which needs attention. There are long term and damaging effects of psychological stress due to negative events such as this pandemic in children who can face high levels of stress, anxiety, and depression as a result of it [4]. If properly supported by the healthcare professionals and the members of their multidisciplinary care team, children and their families can appropriately overcome any distress.

Such support can be provided through the telehealth measures including telerehabilitation and teleconsultation as these could be appropriate and helpful ways to address the gap in the care of such children in the current situations of lockdown [5].

\section{Acknowledgement}

The authors would like to acknowledge all the class teachers and the parents of the children attending Al Noor Centre for Persons with Disabilities, Dubai, United Arab Emirates.

\section{Competing Interest}

None.

\section{Funding}

Not needed for the study.

\section{References}

1. Lee J (2020) Mental health effects of school closures during COVID-19. Lancet Child Adolesc Health 4: 421.

2. Bao Y, Sun Y, Meng S, et al. (2020) 2019-nCoV epidemic: Address mental health care to empower society. The Lancet 22: e37-e38.

3. Cacioppo M, Bouvier S, Bailly R, et al. (2020) Emerging health challenges for children with physical disabilities and their parents during the COVID-19 pandemic: The ECHO French survey. Ann Phys Rehabil Med 18: 101429.

4. Wang G, Zhang Y, Zhao J, et al. (2020) Mitigate the effects of home confinement on children during the COVID-19 outbreak. The Lancet 395: 945-947.

5. Fazzi E, Galli J (2020) New clinical needs and strategies for care in children with neurodisability during COVID-19. Dev Med Child Neurol 62: 879-880. 